\title{
CP violation effect in long-baseline neutrino oscillation in the four-neutrino model
}

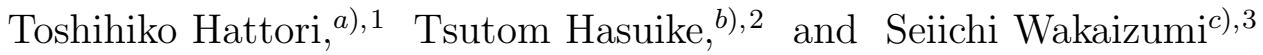 \\ a) Institute of Theoretical Physics, University of Tokushima, Tokushima 770-8502, Japan \\ b) Department of Physics, Anan College of Technology, Anan 774-0017, Japan \\ ${ }^{c}$ School of Medical Sciences, University of Tokushima, Tokushima 770-8509, Japan
}

\begin{abstract}
We investigate $\mathrm{CP}$-violation effect in the long-baseline neutrino oscillation in the four-neutrino model with mass scheme of the two nearly degenerate pairs separated with the order of $1 \mathrm{eV}$, by using the data from the solar neutrino deficit, the atmospheric neutrino anomaly and the LSND experiments along with the other accelerator and reactor experiments. By use of the most general parametrization of the mixing matrix with six angles and six phases, we show that the genuine CP-violation effect could attain as large as 0.3 for $\Delta P\left(\nu_{\mu} \rightarrow \nu_{\tau}\right) \equiv P\left(\nu_{\mu} \rightarrow\right.$ $\left.\nu_{\tau}\right)-P\left(\overline{\nu_{\mu}} \rightarrow \overline{\nu_{\tau}}\right)$ and that the matter effect is negligibly small such as at most 0.01 for $\Delta P\left(\nu_{\mu} \rightarrow \nu_{\tau}\right)$ for $\Delta m^{2}=(1-5) \times 10^{-3} \mathrm{eV}^{2}$, which is the mass-squared difference relevant to the long-baseline oscillation.
\end{abstract}

\footnotetext{
${ }^{1}$ e-mail: hattori@ias.tokushima-u.ac.jp

${ }^{2}$ e-mail: hasuike@anan-nct.ac.jp

${ }^{3}$ e-mail: wakaizum@medsci.tokushima-u.ac.jp
} 


\section{Introduction}

It has long been assumed that neutrinos are massless. However, since the atmospheric neutrino anomaly was discovered by several experimental Collaborations [1] and was affirmatively confirmed by the Super-Kamiokande[2], people have come to think through the neutrino oscillation interpretation for the anomaly that neutrinos seem to have a certain amount of mass. Together with the solar neutrino deficit [3], the anomaly has been analyzed in the three-neutrino model[4] and two typical mass scales have been derived for the neutrino mass-squared difference; $\Delta m_{\text {atm }}^{2}=(0.5-6) \times 10^{-3} \mathrm{eV}^{2}$ with a large mixing angle of $\sin ^{2} 2 \theta_{\mathrm{atm}}>0.82$ as the $\nu_{\mu} \rightarrow \nu_{\tau}$ oscillation from the atmospheric neutrino anomaly [2] and $\Delta m_{\text {solar }}^{2}=$ $\left(10^{-11}-10^{-5}\right) \mathrm{eV}^{2}$, a large range depending on the three solutions of the vacuum oscillation and the MSW solutions in the matter with small- and large-angle mixings from the solar neutrino deficit[5].

As in the quark sector, CP violation would be a characteristic feature in the three-neutrino model. It has been shown[6][0] [8] [9] by using the constraints on the mixing matrix elements obtained from the analyses of these anomalies along with the results from the other accelerator and reactor experiments that the $\mathrm{CP}$ violation effect, defined as a difference of the oscillation probabilities between the neutrino and the antineutrino, is typically $1-3 \%$ even in the long-baseline neutrino oscillations, depending on the assumed mass hierarchies.

On the other hand, sterile neutrinos were considered in the context of neutrino oscillations [10] [11. After that, a four-neutrino model of the ordinary three active neutrinos and one sterile neutrino was introduced with a mass pattern of two nearly degenerate pairs separated with a mass gap of the order of $1 \mathrm{eV}$ motivated from the hot dark matter [12] and then, by using the only one possible positive evidence from the terrestrial LSND experiments on the oscillations $\nu_{\mu} \rightarrow \nu_{e}$ and

$\overline{\nu_{\mu}} \rightarrow \overline{\nu_{e}}$ [13], which suggest the mass scale of $\Delta m_{\mathrm{LSND}}^{2}=(0.3-2.2) \mathrm{eV}^{2}$, the 
four-neutrino model with the same mass pattern as the above is studied [14-20]. In this model, a sizable $\mathrm{CP}$ violation effect is shown to be possible in the longbaseline experiments [15] 21], and different magnitudes of the probability difference between the CP-conjugate channels are expected in between the three-neutrino model and the four-neutrino model [22] by using the most general parametrization of the mixing matrix [20]. And, some features of CP asymmetry defined as the normalized probability difference are discussed in the long-baseline experiments at a neutrino factory 23$]$.

We will investigate the CP violation effect in the long-baseline neutrino oscillations numerically in more detail in the four-neutrino model with mass scheme of the two nearly degenerate pairs separated with the order of $1 \mathrm{eV}$ by using the most general parametrization of the mixing matrix, and in addition we will study the matter effect in the four-neutrino model.

The paper is organized as follows. In Sec. II the four-neutrino model we use here is presented and the expressions of the difference of oscillation probabilities between the CP-conjugate channels are given both in the exact form and in the approximate forms relevant to the short-baseline and the long-baseline neutrino oscillations for the neutrino mass scheme mentioned above. In Sect. III constraints on the neutrino mixing matrix are derived by using the solar neutrino deficit, atmospheric neutrino anomaly, Bugey reactor experiment, CHOOZ experiment, LSND experiments, CHORUS and NOMAD experiments and the other accelerator and reactor experiments. In Sect. IV the most general parametrization of the mixing matrix is adopted to obtain the constraints on the mixing angles and phases from the ones on the mixing matrix derived in Sect. III. And then, CP-violation in the long-baseline neutrino oscillation is investigated on the basis of these constraints. The behavior of the oscillation probability differences is analyzed in detail with respect to the two relevant phases of the mixing matrix and 
$\Delta m^{2} / E$. The matter effect is shown to be negligibly small in the four-neutrino model with the mass scheme adopted here. Finally, Sect. V is devoted to the conclusion.

\section{The four-neutrino model}

In order to consider the solar neutrino deficit, the atmospheric neutrino anomaly and the LSND experiment, we will take the four-neutrino model with the three ordinary active neutrinos and one sterile neutrino with three different scales of the neutrino mass-squared difference, $\Delta m_{\text {solar }}^{2}=\left(10^{-11}-10^{-5}\right) \mathrm{eV}^{2}, \Delta m_{\text {atm }}^{2}=$ $\left(10^{-3}-10^{-2}\right) \mathrm{eV}^{2}$ and $\Delta m_{\mathrm{LSND}}^{2}=(0.3-10) \mathrm{eV}^{2}$.

Under the neutrino oscillation hypothesis [24] 25], the flavor eigenstates of neutrinos are the mixtures of mass eigenstates with masses $m_{i}(i=1,2,3,4)$ as follows,

$$
\nu_{\alpha}=\sum_{i=1}^{4} U_{\alpha i} \nu_{i}, \quad \alpha=e, \mu, \tau, s
$$

where $\nu_{e}, \nu_{\mu}$ and $\nu_{\tau}$ are the ordinary neutrinos and $\nu_{s}$ is the sterile neutrino, and $U$ is the unitary mixing matrix. The neutrino oscillation probability of $\nu_{\alpha} \rightarrow \nu_{\beta}$ in vacuum is given in the usual manner in the four-neutrino model by

$P\left(\nu_{\alpha} \rightarrow \nu_{\beta}\right)=\delta_{\alpha \beta}-4 \sum_{k>j} \operatorname{Re}\left(U_{\alpha k}^{*} U_{\alpha j} U_{\beta j}^{*} U_{\beta k}\right) \sin ^{2} \Delta_{k j}+2 \sum_{k>j} \operatorname{Im}\left(U_{\alpha k}^{*} U_{\alpha j} U_{\beta j}^{*} U_{\beta k}\right) \sin 2 \Delta_{k j}$,

where $\Delta_{k j} \equiv \Delta m_{k j}^{2} L /(4 E), L$ being the distance from the neutrino source and $E$ the energy of neutrino. The oscillation probability for the antineutrinos is given by the exchange of $U \leftrightarrow U^{*}$ in Eq.(21). And, the probability difference between CP-conjugate channels given by

$$
\begin{aligned}
\Delta P_{\alpha \beta} & \equiv P\left(\nu_{\alpha} \rightarrow \nu_{\beta}\right)-P\left(\overline{\nu_{\alpha}} \rightarrow \overline{\nu_{\beta}}\right) \\
& =4 \sum_{k>j} \operatorname{Im}\left(U_{\alpha k}^{*} U_{\alpha j} U_{\beta j}^{*} U_{\beta k}\right) \sin 2 \Delta_{k j}
\end{aligned}
$$

is a direct measure of the genuine $\mathrm{CP}$-violation effect in the neutrino oscillation in vacuum[26]. 
The four neutrino masses should be devided into two pairs of close masses separated by a gap of about $1 \mathrm{eV}$ in order to accomodate with the solar and atmospheric neutrino deficits and the LSND experiments along with the other results from the accelerator and reactor experiments on the neutrino oscillation. There are the following two schemes for that mass pattern; (i) $\Delta m_{\text {solar }}^{2} \equiv \Delta m_{21}^{2} \ll$ $\Delta m_{\mathrm{atm}}^{2} \equiv \Delta m_{43}^{2} \ll \Delta m_{\mathrm{LSND}}^{2} \equiv \Delta m_{32}^{2}$, and (ii) $\Delta m_{\mathrm{solar}}^{2} \equiv \Delta m_{43}^{2} \ll \Delta m_{\mathrm{atm}}^{2} \equiv$ $\Delta m_{21}^{2} \ll \Delta m_{\text {LSND }}^{2} \equiv \Delta m_{32}^{2}$, where $\Delta m_{k j}^{2} \equiv m_{k}^{2}-m_{j}^{2}$. We will adopt the first scheme in the following analyses, and the second scheme can be attained only through the exchange of indices $(1,2) \leftrightarrow(3,4)$ in the following various expressions such as the oscillation probabilities. In the first scheme, the measure of $\mathrm{CP}$ violation in the neutrino oscillation in vacuum is given for the short-baseline experiment $(L / E \sim 1[\mathrm{~km} / \mathrm{GeV}])$ as follows,

$$
\begin{aligned}
\Delta P_{\alpha \beta} \simeq & 4\left[\operatorname{Im}\left(U_{\alpha 3}^{*} U_{\alpha 2} U_{\beta 2}^{*} U_{\beta 3}\right)+\operatorname{Im}\left(U_{\alpha 3}^{*} U_{\alpha 1} U_{\beta 1}^{*} U_{\beta 3}\right)\right. \\
& \left.+\operatorname{Im}\left(U_{\alpha 4}^{*} U_{\alpha 2} U_{\beta 2}^{*} U_{\beta 4}\right)+\operatorname{Im}\left(U_{\alpha 4}^{*} U_{\alpha 1} U_{\beta 1}^{*} U_{\beta 4}\right)\right] \sin 2 \Delta_{32}
\end{aligned}
$$

since $\Delta_{21}$ and $\Delta_{43} \ll 1$, and $\Delta_{41}, \Delta_{42}, \Delta_{31}, \Delta_{32} \simeq 1 . \Delta P_{\alpha \beta}$ in Eq.(何) is zero due to the unitarity of the mixing matrix $U$. So, $\mathrm{CP}$ violation is negligibly small in the short-baseline oscillation experiments in the four-neutrino model.

On the other hand, for the long-baseline experiment $(L / E=100-1000$ $[\mathrm{km} / \mathrm{GeV}])$ the probability difference in vacuum is given as follows,

$$
\Delta P_{\alpha \beta} \simeq 4 \operatorname{Im}\left(U_{\alpha 4}^{*} U_{\alpha 3} U_{\beta 3}^{*} U_{\beta 4}\right) \sin 2 \Delta_{43}
$$

since $\Delta_{21} \ll 1, \Delta_{41}, \Delta_{42}, \Delta_{31}, \Delta_{32} \gg 1$, and $\Delta_{43} \sim 1$. There are six $\Delta P_{\alpha \beta}$ 's, that is, $\Delta P_{\mu e}, \Delta P_{e \tau}, \Delta P_{\mu \tau}, \Delta P_{e s}, \Delta P_{\mu s}$, and $\Delta P_{\tau s}$. Three of these six $\Delta P_{\alpha \beta}$ 's are independent due to the unitarity of $U$ for the approximate expression of $\Delta P_{\alpha \beta}$ in Eq.(5) as well as for the exact expression in Eq.(3). 


\section{Constraints on the mixing matrix $U$}

In order to numerically calculate the oscillation probability differences $\Delta P_{\alpha \beta}$, we will derive the constraints on the mixing matrix $U$ from the solar neutrino deficit, atmospheric neutrino anomaly, LSND experiments and the other terrestrial oscillation experiments using the accelerators and reactors.

(i) Solar neutrino deficit

Since $\Delta_{21} \sim 1$ and all the other five $\Delta_{k j}$ 's are enormously larger than 1 , the survival probability of $\nu_{e}$ is given from Eq.(2) by

$$
\begin{aligned}
P_{\text {solar }}\left(\nu_{e} \rightarrow \nu_{e}\right) \simeq & 1-4\left|U_{e 1}\right|^{2}\left|U_{e 2}\right|^{2} \sin ^{2} \Delta_{21}-2\left|U_{e 3}\right|^{2}\left(1-\left|U_{e 3}\right|^{2}-\left|U_{e 4}\right|^{2}\right) \\
& -2\left|U_{e 4}\right|^{2}\left(1-\left|U_{e 4}\right|^{2}\right),
\end{aligned}
$$

where the unitarity of $U$ is used. For the solar neutrino deficit, there are three different kinds of solutions, that is, the vacuum solution and the MSW solutions with small and large angle mixings, and a unique solution is not yet found, so that we will not use this deficit in order to obtain the constraints.

(ii) Atmospheric neutrino anomaly

Since $\Delta_{21} \ll 1, \Delta_{43} \sim 1$ and $\Delta_{41}, \Delta_{42}, \Delta_{31}, \Delta_{32} \gg 1$, the survival probability of $\nu_{\mu}$ is given by

$P_{\mathrm{atm}}\left(\nu_{\mu} \rightarrow \nu_{\mu}\right) \simeq 1-4\left|U_{\mu 3}\right|^{2}\left|U_{\mu 4}\right|^{2} \sin ^{2} \Delta_{43}-2\left(\left|U_{\mu 1}\right|^{2}+\left|U_{\mu 2}\right|^{2}\right)\left(1-\left|U_{\mu 1}\right|^{2}-\left|U_{\mu 2}\right|^{2}\right)$

By using the data from the Super-Kamiokande experiments, that is, $\sin ^{2} 2 \theta_{\text {atm }}>$

0.82 for $5 \times 10^{-4}<\Delta m_{\text {atm }}^{2}<6 \times 10^{-3} \mathrm{eV}^{2}$, and expecting from this data that $\left|U_{\mu 1}\right|^{2}+\left|U_{\mu 2}\right|^{2} \ll 1$, the following constraint is obtained,

$$
\left|U_{\mu 3}\right|^{2}\left|U_{\mu 4}\right|^{2}>0.205
$$

(iii) The Bugey experiment 27] (including Krasnoyarsk 28, CDHS 29] and CCFR 30] experiments) 
By being typically represented by the Bugey reactor experiment with $L / E=3-20$ $[\mathrm{m} / \mathrm{MeV}$ or $\mathrm{km} / \mathrm{GeV}]$, since $\Delta_{21} \ll 1, \Delta_{43} \ll 1$ and $\Delta_{41}, \Delta_{42}, \Delta_{31}, \Delta_{32} \sim 1$, the survival probability of $\overline{\nu_{e}}$ is given by

$$
P_{\text {Bugey }}\left(\overline{\nu_{e}} \rightarrow \overline{\nu_{e}}\right) \simeq 1-4\left(\left|U_{e 3}\right|^{2}+\left|U_{e 4}\right|^{2}\right)\left(1-\left|U_{e 3}\right|^{2}-\left|U_{e 4}\right|^{2}\right) \sin ^{2} \Delta_{32} .
$$

If we use the data from the Bugey experiment conservatively, that is, $\sin ^{2} 2 \theta_{\text {Bugey }}<$ 0.1 for $0.1<\Delta m^{2}<1 \mathrm{eV}^{2}$, the following constraint is obtained,

$$
\left|U_{e 3}\right|^{2}+\left|U_{e 4}\right|^{2}<0.025 .
$$

(iv) The CHOOZ experiment 31

This experiment is the first long-baseline reactor experiment, since $L \sim 1 \mathrm{~km}$ and $E \sim 3 \mathrm{MeV}$ so that $L / E \sim 300[\mathrm{~km} / \mathrm{GeV}]$. Therefore, $\Delta_{21} \ll 1, \Delta_{43} \sim 1$ and $\Delta_{41}, \Delta_{42}, \Delta_{31}, \Delta_{32} \gg 1$, and the survival probability of $\overline{\nu_{e}}$ is given by

$P_{\mathrm{CHOOZ}}\left(\overline{\nu_{e}} \rightarrow \overline{\nu_{e}}\right) \simeq 1-4\left|U_{e 3}\right|^{2}\left|U_{e 4}\right|^{2} \sin ^{2} \Delta_{43}-2\left(\left|U_{e 3}\right|^{2}+\left|U_{e 4}\right|^{2}\right)\left(1-\left|U_{e 3}\right|^{2}-\left|U_{e 4}\right|^{2}\right)$.

By using the data from the CHOOZ experiment, that is, $\sin ^{2} 2 \theta_{\mathrm{CHOOZ}}<0.12$ for $3 \times 10^{-3}<\Delta m^{2}<1.0 \times 10^{-2} \mathrm{eV}^{2}$ and adopting Eq.(10), the following constraint is obtained,

$$
4\left|U_{e 3}\right|^{2}\left|U_{e 4}\right|^{2}<0.12 \text {. }
$$

If we use, however, the constraint of Eq.(10) and the unequality of $2\left|U_{e 3}\right|\left|U_{e 4}\right| \leq$ $\left|U_{e 3}\right|^{2}+\left|U_{e 4}\right|^{2}$, a constraint $4\left|U_{e 3}\right|^{2}\left|U_{e 4}\right|^{2}<6.3 \times 10^{-4}$ is obtained so that Eq.(12) is included in the constraint from the Bugey experiment.

(v) The LSND experiments[13]

This experiment is of the short baseline, $L / E=0.5-1[\mathrm{~m} / \mathrm{MeV}]$. Since $\Delta_{21} \ll$ $1, \Delta_{43} \ll 1$ and $\Delta_{41}, \Delta_{42}, \Delta_{31}, \Delta_{32} \sim 1$, the oscillation probability of $\nu_{\mu} \rightarrow \nu_{e}$ is expressed as follows,

$$
\begin{aligned}
P_{\mathrm{LSND}}\left(\nu_{\mu} \rightarrow \nu_{e}\right) & \simeq-4 \operatorname{Re}\left[\left(U_{\mu 3}^{*} U_{e 3}+U_{\mu 4}^{*} U_{e 4}\right)\left(U_{\mu 1} U_{e 1}^{*}+U_{\mu 2} U_{e 2}^{*}\right)\right] \sin ^{2} \Delta_{32} \\
& =4\left|U_{\mu 3}^{*} U_{e 3}+U_{\mu 4}^{*} U_{e 4}\right|^{2} \sin ^{2} \Delta_{32},
\end{aligned}
$$


where the unitarity of $U$ is used. By using the data from the LSND experiments, that is, $\sin ^{2} 2 \theta_{\mathrm{LSND}}=1.5 \times 10^{-3}-1.0 \times 10^{-1}$ for $0.3<\Delta m_{\mathrm{LSND}}^{2}<2.2 \mathrm{eV}^{2}$, the following constraint is obtained,

$$
\left|U_{\mu 3}^{*} U_{e 3}+U_{\mu 4}^{*} U_{e 4}\right|=0.02-0.16 .
$$

(vi) The CHORUS[32] and NOMAD [33] experiments

These experiments are also the short baseline ones searching for the $\nu_{\mu} \rightarrow \nu_{\tau}$ oscillation, $L / E=0.02-0.03[\mathrm{~km} / \mathrm{GeV}]$. Since $\Delta_{21} \ll 1, \Delta_{43} \ll 1$ and $\Delta_{41}, \Delta_{42}, \Delta_{31}, \Delta_{32} \simeq$ $10^{-2}-10^{-1}$, the oscillation probability is given by

$$
P_{\mathrm{CHORUS} / \mathrm{NOMAD}}\left(\nu_{\mu} \rightarrow \nu_{\tau}\right) \simeq 4\left|U_{\mu 3}^{*} U_{\tau 3}+U_{\mu 4}^{*} U_{\tau 4}\right|^{2} \sin ^{2} \Delta_{32} .
$$

By using the data from the latest NOMAD experiment, $\sin ^{2} 2 \theta_{\text {NOMAD }}<0.3$ for $\Delta m^{2}<2.2 \mathrm{eV}^{2}$, the following constraint is obtained,

$$
\left|U_{\mu 3}^{*} U_{\tau 3}+U_{\mu 4}^{*} U_{\tau 4}\right|<0.28
$$

Among the above-mentioned six typical phenomena and experiments, the useful constraints are of Eqs. (8), (10), (14) and (16).

\section{CP violation in the neutrino oscillations}

In this section, by using the constraints obtained in the previous section, we will numerically investigate the $\mathrm{CP}$ violation effects in the long-baseline neutrino oscillation experiments in the four-neutrino model described in Sect. II.

We adopt the most general parametrization of the mixing matrix $U$ for Majorana neutrinos [20], which includes six mixing angles and six phases. The expression of the matrix is too complicated to write it down here. So, we cite only the matrix elements which are useful for the following numerical analyses; $U_{e 1}=c_{01} c_{02} c_{03}, U_{e 2}=c_{02} c_{03} s_{d 01}^{*}, U_{e 3}=c_{03} s_{d 02}^{*}, U_{e 4}=s_{d 03}^{*}, U_{\mu 3}=-s_{d 02}^{*} s_{d 03} s_{d 13}^{*}+$

$c_{02} c_{13} s_{d 12}^{*}, U_{\mu 4}=c_{03} s_{d 13}^{*}, U_{\tau 3}=-c_{13} s_{d 02}^{*} s_{d 03} s_{d 23}^{*}-c_{02} s_{d 12}^{*} s_{d 13} s_{d 23}^{*}+c_{02} c_{12} c_{23}$, and 
$U_{\tau 4}=c_{03} c_{13} s_{d 23}^{*}$, where $c_{i j} \equiv \cos \theta_{i j}$ and $s_{d i j} \equiv s_{i j} \mathrm{e}^{\mathrm{i} \delta_{i j}} \equiv \sin \theta_{i j} \mathrm{e}^{\mathrm{i} \delta_{i j}}$ [20], and $\theta_{01}, \theta_{02}, \theta_{03}, \theta_{12}, \theta_{13}, \theta_{23}$ are the six angles and $\delta_{01}, \delta_{02}, \delta_{03}, \delta_{12}, \delta_{13}, \delta_{23}$ are the six phases. As stated in Sect. II, three of the six oscillation probability differences are independent so that only three of the six phases are determined by the measurements of the $\mathrm{CP}$ violation effect in the neutrino oscillations. In this sense, our analyses apply both to the Dirac and Majorana neutrinos 22.

On the basis of this parametrization, we obtain the constraints on the mixing angles and phases by using the constraints on the mixing matrix elements derived in the previous section. First, the constraint of Eq.(10) leads to

$$
c_{03}^{2} s_{02}^{2}+s_{03}^{2}<0.025 .
$$

This unequality means at least $s_{02}^{2}, s_{03}^{2}<0.025$. The next constraint of Eq.(8) of

$$
\left|-s_{02} s_{03} s_{13} \mathrm{e}^{-\mathrm{i}\left(\delta_{02}-\delta_{03}+\delta_{13}\right)}+c_{02} c_{13} s_{12} \mathrm{e}^{-\mathrm{i} \delta_{12}}\right|^{2} c_{03}^{2} s_{13}^{2}>0.205
$$

leads to

$$
s_{12}^{2} c_{13}^{2} s_{13}^{2}>0.205
$$

due to the smallness of $s_{02}$ and $s_{03}$. The third constraint of Eq.(14) gives the following expression,

$$
\left|c_{02} s_{02} c_{03} s_{12} c_{13}+c_{02}^{2} c_{03} s_{03} s_{13} \mathrm{e}^{\mathrm{i} \delta_{1}}\right|=0.02-0.16,
$$

where $\delta_{1} \equiv \delta_{02}-\delta_{03}-\delta_{12}+\delta_{13}$. This constraint proves not to bring any constraint on the phase $\delta_{1}$, if we use Eqs.(17) and (19). The fourth constraint of Eq.(16) is expressed as

$$
\begin{aligned}
& \mid \quad c_{02}^{2} c_{12} s_{12} c_{13} c_{23}-c_{02} s_{02} s_{03} s_{12} c_{13}^{2} s_{23} \mathrm{e}^{-\mathrm{i}\left(\delta_{1}+\delta_{2}\right)}-c_{02} s_{02} s_{03} c_{12} s_{13} c_{23} \mathrm{e}^{\mathrm{i} \delta_{1}} \\
& \quad+c_{13} s_{13} s_{23}\left(c_{03}^{2}-c_{02}^{2} s_{12}^{2}+s_{02}^{2} s_{03}^{2}\right) \mathrm{e}^{-\mathrm{i} \delta_{2}} \mid<0.28,
\end{aligned}
$$

where $\delta_{2} \equiv \delta_{12}-\delta_{13}+\delta_{23}$. By using Eqs. (17) and (19), no constraint on $\delta_{1}$, and the fact of the large angle mixing in $\nu_{\mu} \rightarrow \nu_{\tau}$ oscillation for the atmospheric 
neutrino anomaly which leads to the nearly maximal mixing in the angle $\theta_{23}$, the constraint of Eq. (21) gives no constraint to the phase $\delta_{2}$.

So, in summary, we derive the two constraints of Eqs. (17) and (19) on the mixing angles and no constraints on the two phases of $\delta_{1}$ and $\delta_{2}$.

Using these two constraints on the mixing angles, we will calculate the differences of the oscillation probabilities between the CP-conjugate channels for the long-baseline neutrino oscillations. As stated before, only three of the six probability differences among the four neutrinos are independent so that three of the six phases are relevant here. However, only two phases dominantly affect the differences as is shown by the leading terms relevant to the long-baseline oscillation, which are given in the following,

$$
\begin{aligned}
\Delta P_{\mu e} \simeq & 4 c_{03}^{2} c_{02} s_{02} s_{03} s_{12} c_{13} s_{13} \sin \delta_{1} \sin 2 \Delta_{43} \\
\Delta P_{e \tau} \simeq & 4 c_{03}^{2} c_{02} s_{02} s_{03} c_{13} s_{23}\left[-c_{12} c_{23} \sin \left(\delta_{1}+\delta_{2}\right)+s_{12} s_{13} s_{23} \sin \delta_{1}\right] \sin 2 \Delta_{43}, \\
\Delta P_{\mu \tau} \simeq & 4 c_{03}^{2} c_{02} c_{13} s_{13} s_{23}\left[c_{02} c_{12} s_{12} c_{13} c_{23} \sin \delta_{2}+s_{02} s_{03} s_{12} s_{23} \sin \delta_{1}\right. \\
& \left.-s_{02} s_{03} c_{12} s_{13} c_{23} \sin \left(\delta_{1}+\delta_{2}\right)\right] \sin 2 \Delta_{43}, \\
\Delta P_{e s} \simeq & 4 c_{03}^{2} c_{02} s_{02} s_{03} c_{13} c_{23}\left[c_{12} s_{23} \sin \left(\delta_{1}+\delta_{2}\right)+s_{12} s_{13} c_{23} \sin \delta_{1}\right] \sin 2 \Delta_{43}, \\
\Delta P_{\mu s} \simeq & 4 c_{03}^{2} c_{02} c_{13} s_{13} c_{23}\left[c_{02} c_{12} s_{12} c_{13} s_{23} \sin \delta_{2}-s_{02} s_{03} s_{12} c_{23} \sin \delta_{1}\right. \\
& \left.-s_{02} s_{03} c_{12} s_{13} s_{23} \sin \left(\delta_{1}+\delta_{2}\right)\right] \sin 2 \Delta_{43}, \\
\Delta P_{\tau s} \simeq & -4 c_{03}^{2} c_{02} c_{12} c_{13}^{2} c_{23} s_{23}\left[c_{02} s_{12} s_{13} \sin \delta_{2}+s_{02} s_{03} c_{13} \sin \left(\delta_{1}+\delta_{2}\right)\right] \sin 2 \Delta_{43},
\end{aligned}
$$

where $\delta_{1}$ and $\delta_{2}$ are the linear combinations of $\delta_{i j}$ 's as stated before. We take the range of phases as $0 \leq \delta_{i j}<2 \pi$ and the range of mixing angles as $0 \leq \theta_{i j} \leq \pi$ so that $s_{i j}$ 's can be taken only positive and $c_{i j}$ 's can be taken both positive and negative. Since Eq. (17) means that the angles $\theta_{02}$ and $\theta_{03}$ are very small and Eq. (19) leads to $s_{12}^{2} \sin ^{2} 2 \theta_{13}>0.82$ which means that $s_{12}$ is in the range of $0.9 \leq s_{12} \leq 1.0$ and the angle $\theta_{13}$ is around $\pi / 4, \Delta P_{\mu e}$ and $\Delta P_{e \tau}$ are expected from Eq.(22) to be very small and $\Delta P_{\mu \tau}$ is to be able to take a sizable magnitude. 
Table 1: Phase $\delta_{1}$-dependence of the oscillation probabilities $P_{\alpha \beta}$ and their differences $\Delta P_{\alpha \beta}$ for the long-baseline experiment. $P_{\alpha \beta} \equiv P\left(\nu_{\alpha} \rightarrow \nu_{\beta}\right)$ and $\Delta P_{\alpha \beta} \equiv P\left(\nu_{\alpha} \rightarrow \nu_{\beta}\right)-P\left(\overline{\nu_{\alpha}} \rightarrow \overline{\nu_{\beta}}\right) . \delta_{1}$ is in degree.

\begin{tabular}{rcccccc}
\hline$\delta_{1}$ & $P_{\mu e}$ & $P_{e \tau}$ & $P_{\mu \tau}$ & \multicolumn{1}{c}{$\Delta P_{\mu e}$} & \multicolumn{1}{c}{$\Delta P_{e \tau}$} & $\Delta P_{\mu \tau}$ \\
\hline $0^{\circ}$ & 0.032 & 0.004 & 0.148 & 0.000 & -0.007 & -0.269 \\
$45^{\circ}$ & 0.036 & 0.008 & 0.148 & 0.015 & 0.002 & -0.277 \\
$90^{\circ}$ & 0.032 & 0.015 & 0.147 & 0.022 & 0.011 & -0.283 \\
$135^{\circ}$ & 0.022 & 0.021 & 0.147 & 0.015 & 0.013 & -0.282 \\
$180^{\circ}$ & 0.011 & 0.022 & 0.148 & 0.000 & 0.007 & -0.275 \\
$225^{\circ}$ & 0.006 & 0.018 & 0.148 & -0.015 & -0.002 & -0.267 \\
$270^{\circ}$ & 0.011 & 0.011 & 0.148 & -0.022 & -0.011 & -0.261 \\
$315^{\circ}$ & 0.021 & 0.005 & 0.148 & -0.015 & -0.013 & -0.262 \\
\hline
\end{tabular}

Table 2: Phase $\delta_{2}$-dependence of the oscillation probabilities $P_{\alpha \beta}$ and their differences $\Delta P_{\alpha \beta}$ for the long-baseline experiment. $\delta_{2}$ is in degree.

\begin{tabular}{rcccccc}
\hline \multicolumn{1}{c}{$\delta_{2}$} & $P_{\mu e}$ & $P_{e \tau}$ & $P_{\mu \tau}$ & $\Delta P_{\mu e}$ & $\Delta P_{e \tau}$ & $\Delta P_{\mu \tau}$ \\
\hline $0^{\circ}$ & 0.032 & 0.009 & 0.191 & 0.022 & 0.003 & -0.008 \\
$45^{\circ}$ & 0.032 & 0.009 & 0.124 & 0.022 & 0.006 & -0.201 \\
$90^{\circ}$ & 0.032 & 0.015 & 0.147 & 0.022 & 0.011 & -0.283 \\
$135^{\circ}$ & 0.032 & 0.023 & 0.248 & 0.022 & 0.016 & -0.205 \\
$180^{\circ}$ & 0.032 & 0.028 & 0.367 & 0.022 & 0.018 & -0.014 \\
$225^{\circ}$ & 0.032 & 0.028 & 0.433 & 0.022 & 0.016 & 0.179 \\
$270^{\circ}$ & 0.032 & 0.022 & 0.409 & 0.022 & 0.011 & 0.261 \\
$315^{\circ}$ & 0.032 & 0.014 & 0.309 & 0.022 & 0.006 & 0.184 \\
\hline
\end{tabular}

In the following, we calculate the oscillation probabilities $P\left(\nu_{\alpha} \rightarrow \nu_{\beta}\right)$ and their differences $\Delta P_{\alpha \beta}$ by using the rigorous expressions of Eqs. (2) and (3).

The probabilities of $P\left(\nu_{\mu} \rightarrow \nu_{e}\right)$ and $P\left(\overline{\nu_{\mu}} \rightarrow \overline{\nu_{e}}\right)$ as functions of the phase $\delta_{1}$ with $\delta_{2}=\pi / 2$ fixed are shown in Fig.1 and those of $P\left(\nu_{e} \rightarrow \nu_{\tau}\right)$ and $P\left(\overline{\nu_{e}} \rightarrow \overline{\nu_{\tau}}\right)$ as functions of the phase $\delta_{2}$ with $\delta_{1}=\pi / 2$ fixed are shown in Fig.2 for the values of the parameter set of angles and phases; $s_{02}=s_{03}=0.11\left(c_{02}=c_{03}=\right.$ $0.994), s_{12}=0.91\left(c_{12}=0.415\right), s_{13}=0.67\left(c_{13}=0.742\right), s_{01}=s_{23}=1 / \sqrt{2}\left(c_{01}=\right.$ $\left.c_{23}=1 / \sqrt{2}\right)$ and $\delta_{01}=\delta_{02}=\delta_{03}=\delta_{12}=0$, which are chosen so as to give the 
probability differences as large as possible within the parameter ranges allowed by the constaints of Eqs. (17) and (19). The magnitude of these probabilities is at most 0.04 as shown in Figs.1 and 2. Therefore, the probability differences $\Delta P\left(\nu_{\mu} \rightarrow \nu_{e}\right)$ and $\Delta P\left(\nu_{e} \rightarrow \nu_{\tau}\right)$ are at most \pm 0.02 as shown in Fig.3 for the same parameter values. On the other hand, $P\left(\nu_{\mu} \rightarrow \nu_{\tau}\right)$ and $P\left(\overline{\nu_{\mu}} \rightarrow \overline{\nu_{\tau}}\right)$ can rise to as large as $0.40-0.45$ as shown in Fig.4 and $\Delta P\left(\nu_{\mu} \rightarrow \nu_{\tau}\right)$ can attain as large as \pm 0.28 as shown in Fig.5 for the same parameter values. These facts agree with the above-mentioned expectations. The angle $\theta_{23}$-dependence of $\Delta P\left(\nu_{\mu} \rightarrow \nu_{\tau}\right)$ and $\Delta P\left(\nu_{e} \rightarrow \nu_{\tau}\right)$ is shown in Fig.6, where the phases $\delta_{1}$ and $\delta_{2}$ are taken as $\pi / 2$ and the values of the other angles and phases are the same as the above. We display the phase $\delta_{1}$-dependence in Table 1 and the phase $\delta_{2}$-dependence in Table 2 of $P\left(\nu_{\mu} \rightarrow \nu_{e}\right), P\left(\nu_{e} \rightarrow \nu_{\tau}\right), P\left(\nu_{\mu} \rightarrow \nu_{\tau}\right), \Delta P\left(\nu_{\mu} \rightarrow \nu_{e}\right), \Delta P\left(\nu_{e} \rightarrow \nu_{\tau}\right)$ and $\Delta P\left(\nu_{\mu} \rightarrow \nu_{\tau}\right)$.

Here we comment on the matter effect on the oscillation probability difference in the four-neutrino model. By using the Minakata-Nunokawa procedure 8 , the probability difference with the matter effect is expressed for the long-baseline $\nu_{\alpha} \rightarrow \nu_{\beta}$ oscillation in the four-neutrino model with mass scheme of the two nearly degenerate pairs separated with the order of $1 \mathrm{eV}$ as follows,

$$
\begin{aligned}
\Delta P_{\alpha \beta} \simeq \quad & 4 \operatorname{Im}\left(U_{\alpha 4}^{*} U_{\alpha 3} U_{\beta 3}^{*} U_{\beta 4}\right) \cos B_{34} \sin \left(\frac{\Delta m^{2} L}{2 E}\right) \\
& +4 \operatorname{Re}\left(U_{\alpha 4}^{*} U_{\alpha 3} U_{\beta 3}^{*} U_{\beta 4}\right) \sin B_{34} \sin \left(\frac{\Delta m^{2} L}{2 E}\right) \\
& -8 \sum_{j>i} \operatorname{Re}(U U U \delta V)_{\alpha \beta ; i j} \cos ^{2}\left(\frac{B_{i j}}{2}\right) \sin ^{2}\left(\frac{\Delta m_{i j}^{2} L}{4 E}\right),
\end{aligned}
$$

where

$$
\begin{gathered}
B_{i j}=\left(\left|U_{e i}\right|^{2}-\left|U_{e j}\right|^{2}\right) a L+\left(\left|U_{s i}\right|^{2}-\left|U_{s j}\right|^{2}\right) a^{\prime} L \\
(U U U \delta V)_{\alpha \beta ; i j}=U_{\alpha i}^{*} U_{\alpha j} U_{\beta j}^{*} \delta V_{\beta i}+U_{\alpha i}^{*} U_{\alpha j} \delta V_{\beta j}^{*} U_{\beta i} \\
+U_{\alpha i}^{*} \delta V_{\alpha j} U_{\beta j}^{*} U_{\beta i}+\delta V_{\alpha i}^{*} U_{\alpha j} U_{\beta j}^{*} U_{\beta i} .
\end{gathered}
$$


In Eq.(24), the quantity $a$ represents the matter effect for $\nu_{e}$ and we take $a=$ $1.04 \times 10^{-13} \mathrm{eV}$ for the constant matter density of $2.7 \mathrm{~g} / \mathrm{cm}^{3}[\mathbb{8}]$, and $a^{\prime}$ represents the one for $\nu_{s}$ and we take $a^{\prime}=a / 2$ [15]. In Eq.(25), $\delta V_{\alpha i}$ is given [8] by

$$
\delta V_{\alpha i}=\sum_{j \neq i} \frac{2 E}{\Delta m_{i j}^{2}} U_{\alpha j}\left(U_{e j}^{*} U_{e i} a+U_{s j}^{*} U_{s i} a^{\prime}\right) .
$$

In Eq.(23), the first term represents the genuine CP-violation effect corrected by the matter effect, the second term does the CP-violation effect coming from the phase evolution of the neutrino wave function in the matter, and the third one results from the corrections to the mixing matrix $U$ due to the existence of matter 8].

We estimate these matter effects for the $\nu_{\mu} \rightarrow \nu_{\tau}$ oscillation. The first term of Eq. (23) is almost the genuine CP-violation effect, since the magnitude of the matter effect $B_{34}$ is at most $1 \times 10^{-3}$ for the above-mentioned parameter values of the mixing angles and phases. The second term is approximately $0.4 \times 10^{-3}$, since $\operatorname{Re}\left(U_{\mu 4}^{*} U_{\mu 3} U_{\tau 3}^{*} U_{\tau 4}\right) \sim 0.1$ and $\sin B_{34} \sim 1 \times 10^{-3}$. This should be compared with the possible maximum value of the genuine CP-violation effect displayed in Fig.5 and Table 1, that is, $\left|\Delta P\left(\nu_{\mu} \rightarrow \nu_{\tau}\right)\right| \sim 0.3$. The third term of Eq.(23) is expressed as

$$
-8 \sum_{i=1,2, j=3,4} \operatorname{Re}(U U U \delta V)_{\mu \tau ; i j} \sin ^{2}\left(\frac{\Delta M^{2} L}{4 E}\right)-8 \operatorname{Re}(U U U \delta V)_{\mu \tau ; 34} \sin ^{2}\left(\frac{\Delta m^{2} L}{4 E}\right),
$$

since $\cos ^{2}\left(B_{i j} / 2\right) \simeq 1.0$. The coefficient of the first term of Eq. (27) is given by

$$
\begin{aligned}
\sum_{i=1,2, j=3,4} \operatorname{Re} & (U U U \delta V)_{\mu \tau ; i j} \\
= & 4 \frac{2 E a}{\Delta M^{2}} \operatorname{Re}\left[( U _ { \mu 3 } ^ { * } U _ { \tau 3 } + U _ { \mu 4 } ^ { * } U _ { \tau 4 } ) \left\{\left(U_{\mu 3} U_{e 3}^{*}+U_{\mu 4} U_{e 4}^{*}\right)\left(U_{\tau 3}^{*} U_{e 3}+U_{\tau 4}^{*} U_{e 4}\right)\right.\right. \\
& \left.\left.+\frac{1}{2}\left(U_{\mu 3} U_{s 3}^{*}+U_{\mu 4} U_{s 4}^{*}\right)\left(U_{\tau 3}^{*} U_{s 3}+U_{\tau 4}^{*} U_{s 4}\right)\right\}\right]
\end{aligned}
$$

where the relation $a^{\prime}=a / 2$ is used, and the terms with $1 / \Delta m^{2}$ and $1 / \Delta m_{\text {solar }}^{2}$ do not appear due to the symmetry of the mass scheme of the four neutrinos adopted 
in our model. The coefficient of the second term of Eq.(27) is given by

$$
\begin{aligned}
\operatorname{Re} & (U U U \delta V)_{\mu \tau ; 34} \\
& =-\frac{2 E a}{\Delta M^{2}} \operatorname{Re}\left[\{ U _ { \mu 3 } U _ { \mu 4 } ^ { * } ( | U _ { \tau 3 } | ^ { 2 } + | U _ { \tau 4 } | ^ { 2 } ) + U _ { \tau 3 } U _ { \tau 4 } ^ { * } ( | U _ { \mu 3 } | ^ { 2 } + | U _ { \mu 4 } | ^ { 2 } ) \} \left(U_{e 3}^{*} U_{e 4}\right.\right. \\
& \left.\left.+\frac{1}{2} U_{s 3}^{*} U_{s 4}\right)+2 U_{\mu 3}^{*} U_{\mu 4} U_{\tau 3} U_{\tau 4}^{*}\left(\left|U_{e 3}\right|^{2}+\left|U_{e 4}\right|^{2}+\frac{1}{2}\left|U_{s 3}\right|^{2}+\frac{1}{2}\left|U_{s 4}\right|^{2}\right)\right] \\
& +\frac{2 E a}{\Delta m^{2}} \operatorname{Re}\left[\{ U _ { \mu 3 } U _ { \mu 4 } ^ { * } ( | U _ { \tau 3 } | ^ { 2 } - | U _ { \tau 4 } | ^ { 2 } ) + U _ { \tau 3 } U _ { \tau 4 } ^ { * } ( | U _ { \mu 3 } | ^ { 2 } - | U _ { \mu 4 } | ^ { 2 } ) \} \left(U_{e 3}^{*} U_{e 4}\right.\right. \\
& \left.\left.+\frac{1}{2} U_{s 3}^{*} U_{s 4}\right)\right] .
\end{aligned}
$$

The magnitude of Eq.(28) is estimated to be $-0.9 \times 10^{-4}$, and the magnitude of Eq.(29) is to be $1.1 \times 10^{-2}, 0.51 \times 10^{-2}, 1.3 \times 10^{-3}$ for $\Delta m^{2}=(1.0,2.0,5.0) \times 10^{-3}$ $\mathrm{eV}^{2}$, respectively. So, the third term of Eq.(23), that is, Eq.(27) for $\nu_{\mu} \rightarrow \nu_{\tau}$ oscillation is again negligibly small as compared with the possible maximum value of the genuine CP-violation effect. So, the matter effect can be totally neglected in the $\nu_{\mu} \rightarrow \nu_{\tau}$ oscillation in the four-neutrino model with mass scheme of the two nearly degenerate pairs separated with the order of $1 \mathrm{eV}$, as was generally studied for any channels in ref. [22].

As can be seen in Figs.5 and 6 and in Tables 1 and 2, CP violation could be observed as the probability difference between the $\nu_{\mu} \rightarrow \nu_{\tau}$ and $\overline{\nu_{\mu}} \rightarrow \overline{\nu_{\tau}}$ oscillations in the four-neutrino model. So, we show in Figs. 7 and 8 the oscillation probabilities $P\left(\nu_{\mu} \rightarrow \nu_{\tau}\right)$ and $P\left(\overline{\nu_{\mu}} \rightarrow \overline{\nu_{\tau}}\right)$, and their difference $\Delta P\left(\nu_{\mu} \rightarrow \nu_{\tau}\right)$ as functions of $\Delta m^{2} / E\left[\mathrm{eV}^{2} / \mathrm{GeV}\right]$, respectively, for the long-baseline experiments of the MINOS 34] and CERN-ICARUS [35] types, where $E$ is the neutrino energy. In Figs.7 and 8, we have assumed the baseline length as $L=730 \mathrm{~km}$. We can observe from Fig. 8 that if the beam energy is taken as $7 \mathrm{GeV}$, magnitude of the CP violation effect for the $\nu_{\mu} \rightarrow \nu_{\tau}$ channel could attain as large as $|\Delta P| \simeq 0.22$ in the case of $\Delta m^{2} \simeq 3.5 \times 10^{-3} \mathrm{eV}^{2}$. Incidentally, we display the probabilities $P\left(\nu_{\mu} \rightarrow \nu_{e}\right)$ and $P\left(\overline{\nu_{\mu}} \rightarrow \overline{\nu_{e}}\right)$ in Fig.9 and $\Delta P\left(\nu_{\mu} \rightarrow \nu_{e}\right)$ by a dashed curve in Fig. 8. We show in Table 3 the $\Delta m^{2} / E$-dependence of $P\left(\nu_{\mu} \rightarrow \nu_{e}\right), P\left(\nu_{e} \rightarrow\right.$ 
Table 3: $\Delta m^{2} / E$-dependence of the oscillation probabilities $P_{\alpha \beta}$ and their differences $\Delta P_{\alpha \beta}$ for the long-baseline experiment of $L=730 \mathrm{~km} . P_{\alpha \beta} \equiv P\left(\nu_{\alpha} \rightarrow \nu_{\beta}\right)$ and $\Delta P_{\alpha \beta} \equiv P\left(\nu_{\alpha} \rightarrow \nu_{\beta}\right)-P\left(\overline{\nu_{\alpha}} \rightarrow \overline{\nu_{\beta}}\right) . \Delta m^{2} / E$ is in $10^{-3} \mathrm{eV}^{2} / \mathrm{GeV}$.

\begin{tabular}{rcccccc}
\hline$\Delta m^{2} / E$ & $P_{\mu e}$ & $P_{e \tau}$ & $P_{\mu \tau}$ & $\Delta P_{\mu e}$ & $\Delta P_{e \tau}$ & $\Delta P_{\mu \tau}$ \\
\hline 0.1 & 0.023 & 0.007 & 0.068 & 0.004 & 0.002 & -0.051 \\
0.2 & 0.025 & 0.008 & 0.053 & 0.008 & 0.004 & -0.101 \\
0.3 & 0.027 & 0.009 & 0.046 & 0.011 & 0.006 & -0.147 \\
0.4 & 0.028 & 0.010 & 0.047 & 0.014 & 0.007 & -0.188 \\
0.5 & 0.030 & 0.011 & 0.056 & 0.017 & 0.009 & -0.223 \\
0.6 & 0.031 & 0.012 & 0.072 & 0.019 & 0.010 & -0.251 \\
0.7 & 0.032 & 0.013 & 0.096 & 0.021 & 0.010 & -0.270 \\
0.8 & 0.032 & 0.014 & 0.126 & 0.021 & 0.011 & -0.281 \\
0.9 & 0.032 & 0.015 & 0.161 & 0.022 & 0.011 & -0.282 \\
1.0 & 0.032 & 0.016 & 0.201 & 0.021 & 0.010 & -0.274 \\
1.2 & 0.030 & 0.016 & 0.287 & 0.018 & 0.009 & -0.231 \\
1.4 & 0.028 & 0.016 & 0.374 & 0.012 & 0.006 & -0.158 \\
1.6 & 0.024 & 0.014 & 0.450 & 0.005 & 0.002 & -0.064 \\
1.8 & 0.020 & 0.012 & 0.504 & -0.003 & -0.001 & -0.038 \\
2.0 & 0.016 & 0.010 & 0.530 & -0.010 & -0.005 & 0.135 \\
2.5 & 0.011 & 0.005 & 0.460 & -0.021 & -0.011 & 0.279 \\
\hline
\end{tabular}

$\left.\nu_{\tau}\right), P\left(\nu_{\mu} \rightarrow \nu_{\tau}\right), \Delta P\left(\nu_{\mu} \rightarrow \nu_{e}\right), \Delta P\left(\nu_{e} \rightarrow \nu_{\tau}\right)$ and $\Delta P\left(\nu_{\mu} \rightarrow \nu_{\tau}\right)$ for $L=730 \mathrm{~km}$.

\section{Conclusion}

We have derived the constraints on the neutrino mixing matrix by using the data from the solar neutrino deficit, atmospheric neutrino anomaly, LSND oscillation experiments, Bugey experiment and the CHORUS and NOMAD experiments along with the other accelerator and reactor experiments in the four-neutrino model with mass scheme of the two nearly degenerate pairs separated with the order of $1 \mathrm{eV}$. We have used the most general parametrization of the mixing matrix with six mixng angles and six phases applicable to both Majorana and Dirac neutrinos and have obtained the two serious constraints about the four of the six mixing angles and no constraints on the phases. 
By using these constraints, we have calculated the oscillation probabilities of $P\left(\nu_{\mu} \rightarrow \nu_{e}\right), P\left(\nu_{e} \rightarrow \nu_{\tau}\right)$ and $P\left(\nu_{\mu} \rightarrow \nu_{\tau}\right)$ and have investigated $\mathrm{CP}$ violation in the long-baseline neutrino oscillations of $\Delta P\left(\nu_{\mu} \rightarrow \nu_{e}\right), \Delta P\left(\nu_{e} \rightarrow \nu_{\tau}\right)$ and $\Delta P\left(\nu_{\mu} \rightarrow\right.$ $\left.\nu_{\tau}\right)$. The quantity $\Delta P\left(\nu_{\mu} \rightarrow \nu_{\tau}\right)$ is found to be able to attain a value as large as \pm 0.28 due to the large mixing between $\nu_{\mu}$ and $\nu_{\tau}$ and the mass scheme of the four neutrinos and, therefore, it could be observed in the long-baseline experiments. We have shown that the contribution to $\Delta P\left(\nu_{\mu} \rightarrow \nu_{\tau}\right)$ from the matter effect is at most 0.01, 0.005 and 0.001 in magnitude for $\Delta m^{2}=1.0 \times 10^{-3}, 2.0 \times 10^{-3}$ and $5.0 \times 10^{-3} \mathrm{eV}^{2}$, respectively. So,we can conclude that the matter effect is negligibly small in comparison with the possible maximum value of the genuine $\mathrm{CP}$-violation effect of $\left|\Delta P\left(\nu_{\mu} \rightarrow \nu_{\tau}\right)\right|=0.28$.

\section{References}

[1] Kamiokande Collaboration, K.S. Hirata et al., Phys. Lett. B 205, 416(1988); ibid. 280, 146(1992); Y. Fukuda et al., Phys. Lett. B 335, 237 (1994); IMB Collaboration, D Casper et al., Phys. Rev. Lett. 66, 2561(1991); R.BeckerSzendy et al., Phys. Rev. D 46, 3720(1992); R. Becker-Szendy et al., Nucl. Phys. B (Proc. Suppl.) 38, 331(1995); NUSEX Collaboration, M. Aglietta et al., Europhys. Lett. 8, 611(1989); ibid. 15, 559(1991); SOUDAN -2 Collaboration, W.W.M. Allison et al., Phys. Lett. B 391, 491(1997).

[2] Super-Kamiokande Collaboration, Y. Fukuda et al., Phys. Lett. B 433, 9(1998); ibid. 436, 33(1998); Phys. Rev. Lett. 81, 1562(1998); talk by T. Kajita, at Neutrino 98, Takayama, Japan, June 1998.

[3] B.T. Cleveland et al., Nucl. Phys. B (Proc. Suppl.) 38, 47 (1995); Kamiokande Collaboration, Y. Fukuda et al., Phys. Rev. Lett. 77, 1683 
(1996); GALLEX Collaboration, W. Hampel et al., Phys. Lett. B 388, 384 (1996); SAGE Collaboration, J.N. Abdurashitov et al., Phys. Rev. Lett. 77, 4708(1996); Super-Kamiokande Collaboration, talk by Y. Suzuki, at Neutrino 98, Takayama, Japan, June 1998.

[4] G.L. Fogli, E. Lisi and D. Montanino, Phys. Rev. D 49, 3626(1994); S.M. Bilenky and C. Giunti, Phys. Lett. B 444, 379 (1998).

[5] N. Hata and P. Langacker, Phys. Rev. D50, 632 (1994); J.N. Bahcall, P.I. Krastev and A. Yu. Smirnov, Phys. Rev. D 58, 096016(1998).

[6] M. Tanimoto, Phys. Rev. D 55, 322(1997).

[7] J. Arafune and J. Sato, Phys. Rev. D 55, 1653(1997); J. Arafune, M. Koike and J. Sato, Phys. Rev. D 56, 3093(1997).

[8] H. Minakata and H. Nunokawa, Phys. Rev. D 57, 4403(1998).

[9] K.R. Schubert, hep-ph/9902215.

[10] V. Barger, P. Langacker, J. Leveille and S. Pakvasa, Phys. Rev. Lett. 45, 692(1980); V. Barger, N. Deshpande, P.B. Pal, R.J.N. Phillips and K. Whisnant, Phys. Rev. D 43, 1759(1991).

[11] X. Shi, D.N. Schramm and B.D. Fields, Phys. Rev. D 48, 2563(1993); S.M. Bilenky and C. Giunti, Phys. Lett. B 320, 323(1994).

[12] D.O. Caldwell, Perspectives in Neutrinos, Atomic Physics and Gravitation (Editions Frontieres, Gif-sur-Yvette, France, 1993), p. 187; D.O. Caldwell and R.N. Mohapatra, Phys. Rev. D 48, 3259 (1993); J.T. Peltoniemi and J.W.F. Valle, Nucl. Phys. B406, 409(1993); J.R. Primack, J. Holtzman, A. Klypin and D.O. Caldwell, Phys. Rev. Lett. 74, 2160(1995); E.J. Chun, A.S. 
Joshipura and A.Yu. Smirnov, Phys. Lett. B 357, 608(1995); J.J. GomezCadenas and M.C. Gonzalez-Garcia, Z. Phys. C 71, 443(1996).

[13] C. Athanassopoulos et al., Phys. Rev. Lett. 75, 2650 (1995); ibid. 77, 3082(1996); ibid. 81, 1774(1998).

[14] S.M. Bilenky, C. Giunti, C.W. Kim and S.T. Petcov, Phys. Rev. D 54, 4432(1996); S.M. Bilenky, C. Giunti and W. Grimus, Euro. Phys. J. C1, 247(1998); Phys. Rev. D 57, 1920(1998).

[15] S.M. Bilenky, C. Giunti and W. Grimus, Phys. Rev. D 58, 033001(1998).

[16] E. Ma and P. Roy, Phys. Rev. D 52, 4780(1995); A.Yu. Smirnov and M. Tanimoto, Phys. Rev. D 55, 1665(1997).

[17] S. Goswami, Phys. Rev. D 55, 2931(1997).

[18] N. Okada and O. Yasuda, Int. J. Mod. Phys. A 12, 3669(1997).

[19] V. Barger, T.J. Weiler and K. Whisnant, Phys. Lett. B 427, 97(1998); V. Barger, S. Pakvasa, T.J. Weiler and K. Whisnant, Phys. Rev. D 58, 093016(1998).

[20] V. Barger, Y.-B. Dai, K. Whisnant and B.-L. Young, Phys. Rev. D 59, 113010 (1999).

[21] K. Dick, M. Freund, M. Lindner and A. Romanino, Nucl. Phys. B 562, 29(1999).

[22] A. Kalliomäki, J. Maalampi and M. Tanimoto, Phys. Lett. B 469, 179(1999).

[23] A. Donini, M.B. Gavela, P. Hernández and S. Rigolin, hep-ph/9909254, hepph/9910516.

[24] Z. Maki, M. Nakagawa and S. Sakata, Prog. Theor. Phys. 28, 870(1962). 
[25] S.M. Bilenky and B. Pontecorvo, Phys. Reports 41, 225 (1978).

[26] N. Cabibbo, Phys. Lett. 72B, 333(1978); S. Pakvasa, Proc. of the XXth Int. Conf. of High Energy Physics, Madison, Wisconsin, USA, 1980, Part 2, 1165(1980), ed. L. Durand and L.G. Pondram (AIP, New York 1981); V. Barger, K. Whisnant and R.J.N. Phillips, Phys. Rev. Lett. 45, 2084(1980); Phys. Rev. D 23, 2773(1981).

[27] B. Achkar et al., Nucl. Phys. B434, 503(1995).

[28] G.S. Vidyakin et al., Pis'ma Zh. Eksp. Thor. Fiz. 59, 364 (1994); JETP Lett. 59, 390(1994).

[29] CDHS Collaboration, F. Dydak et al., Phys. Lett. B 134, 281(1984).

[30] CCFR Collaboration, I.E. Stockdale et al., Phys. Rev. Lett. 52, 1384 (1984); Z. Phys. C 27, 53(1985); K.S. McFarland et al., Phys. Rev. Lett. 75, 3993(1995).

[31] M. Apollonio et al., Phys. Lett. B 420, 397(1998).

[32] CHORUS Collaboration, E. Eskut et al., Phys. Lett. B 424, 202(1998), ibid. 434, 205(1998).

[33] NOMAD Collaboration, P. Astier et al., Phys. Lett. B 453, 169(1999).

[34] S.G. Wojcicki, Proc. of Neutrino 96, Helsinki, June 1996, ed. K. Enqvist et al., p237 (World Scientific, Singapore, 1997).

[35] ICARUS Collaboration, P. Cennini et al., LNGS-94/99-I, May 1994. 


\section{Figure captions}

Fig.1. The oscillation probability of $\nu_{\mu} \rightarrow \nu_{e}\left(\right.$ solid curve) and $\overline{\nu_{\mu}} \rightarrow \overline{\nu_{e}}$ (dashed curve) with respect to the phase $\delta_{1}$ of the mixing matrix for the long-baseline experiment. The other angles and phases are fixed as $s_{02}=s_{03}=0.11\left(c_{02}=c_{03}=\right.$

$0.994), s_{12}=0.91\left(c_{12}=0.415\right), s_{13}=0.67\left(c_{13}=0.742\right), s_{01}=s_{23}=1 / \sqrt{2}\left(c_{01}=\right.$ $\left.c_{23}=1 / \sqrt{2}\right), \delta_{01}=\delta_{02}=\delta_{03}=\delta_{12}=0$ and $\delta_{2}=\pi / 2$.

Fig.2. The oscillation probability of $\nu_{e} \rightarrow \nu_{\tau}$ (solid curve) and $\overline{\nu_{e}} \rightarrow \overline{\nu_{\tau}}$ (dashed curve) with respect to the phase $\delta_{2}$ of the mixing matrix for the long-baseline experiment. The other angles and phases are the same as in Fig.1 except for $\delta_{1}=\pi / 2$ fixed.

Fig.3. The probability difference $\Delta P\left(\nu_{\mu} \rightarrow \nu_{e}\right)$ (solid curve) and $\Delta P\left(\nu_{e} \rightarrow\right.$ $\left.\nu_{\tau}\right)$ (dashed curve) with respect to the phase $\delta_{1}$ for the long-baseline experiment. The other angles and phases are the same as in Fig.1.

Fig.4. The oscillation probability of $\nu_{\mu} \rightarrow \nu_{\tau}$ (solid curve) and $\overline{\nu_{\mu}} \rightarrow \overline{\nu_{\tau}}$ (dashed curve) with respect to the phase $\delta_{2}$ for the long-baseline experiment. The other angles and phases are the same as in Fig.2.

Fig.5. The probability difference $\Delta P\left(\nu_{\mu} \rightarrow \nu_{\tau}\right)$ (solid curve) and $\Delta P\left(\nu_{e} \rightarrow \nu_{\tau}\right)$ (dashed curve) with respect to the phase $\delta_{2}$ for the long-baseline experiment. The other angles and phases are the same as in Fig.2.

Fig.6. The probability difference $\Delta P\left(\nu_{\mu} \rightarrow \nu_{\tau}\right)$ (solid curve) and $\Delta P\left(\nu_{e} \rightarrow \nu_{\tau}\right)$ (dashed curve) with respect to the angle $\theta_{23}$ for the long-baseline experiment. The other angles and phases are the same as in Fig.1 except for $\delta_{1}=\pi / 2$ fixed. 
Fig.7. The oscillation probability of $\nu_{\mu} \rightarrow \nu_{\tau}$ (solid curve) and $\overline{\nu_{\mu}} \rightarrow \overline{\nu_{\tau}}$ (dashed curve) with respect to $\Delta m^{2} / E$ for the long-baseline experiment with the distance of $L=730 \mathrm{~km}$. The angles and phases are the same as in Fig.1 except for $\delta_{1}=\pi / 2$ fixed.

Fig.8. The probability difference $\Delta P\left(\nu_{\mu} \rightarrow \nu_{\tau}\right)$ (solid curve) and $\Delta P\left(\nu_{\mu} \rightarrow\right.$ $\left.\nu_{e}\right)$ (dashed curve) with respect to $\Delta m^{2} / E$ for the long-baseline experiment with the distance of $L=730 \mathrm{~km}$. The angles and phases are the same as in Fig.7.

Fig.9. The oscillation probability of $\nu_{\mu} \rightarrow \nu_{e}$ (solid curve) and $\overline{\nu_{\mu}} \rightarrow \overline{\nu_{e}}$ (dashed curve) with respect to $\Delta m^{2} / E$ for the long-baseline experiment with the distance of $L=730 \mathrm{~km}$. The angles and phases are the same as in Fig.7. 




Fig. 3 


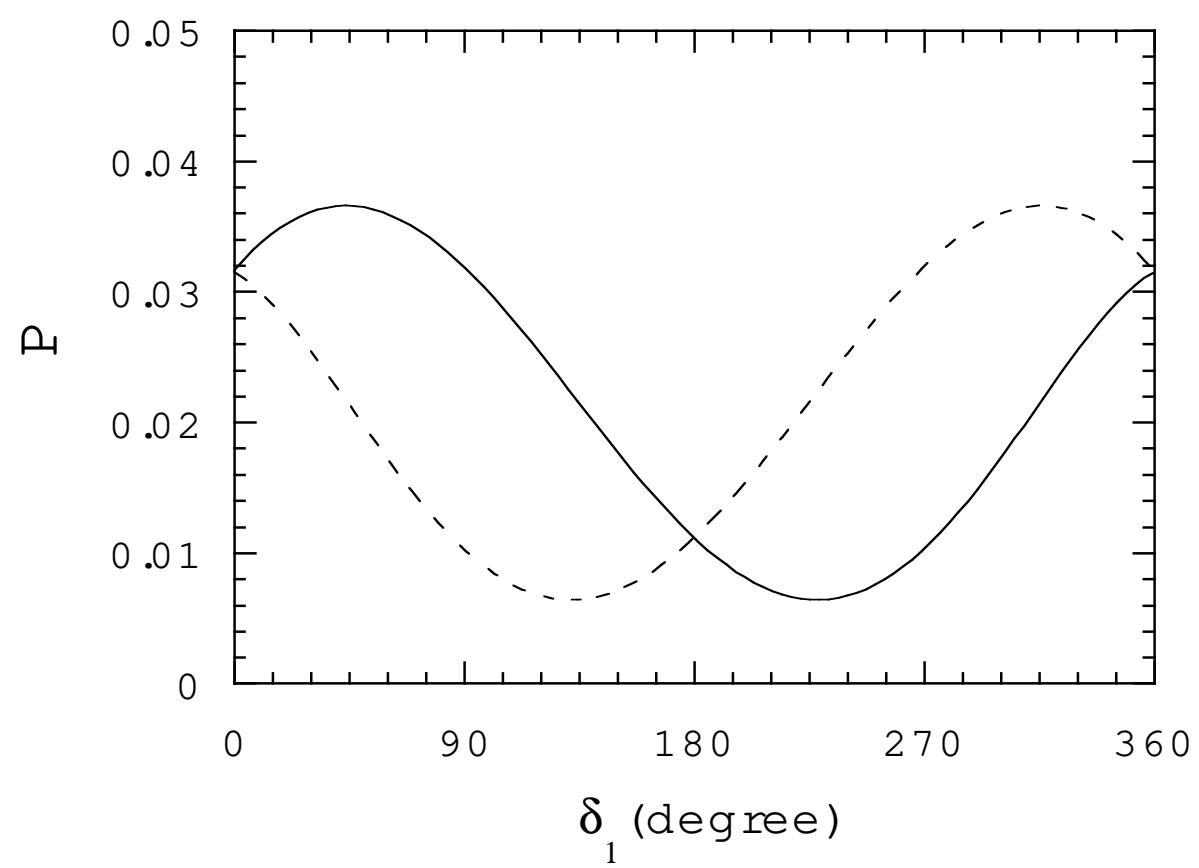

Fig . 1 


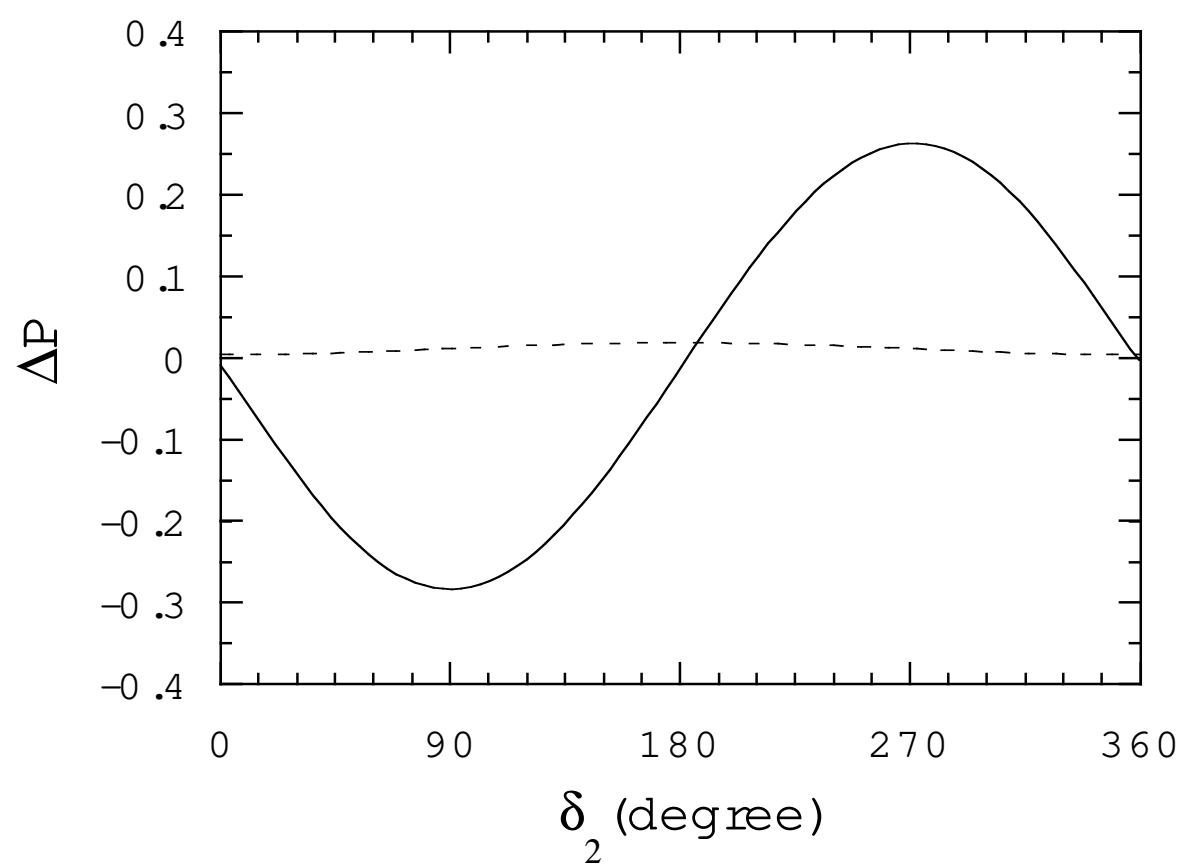

Fig. 5 


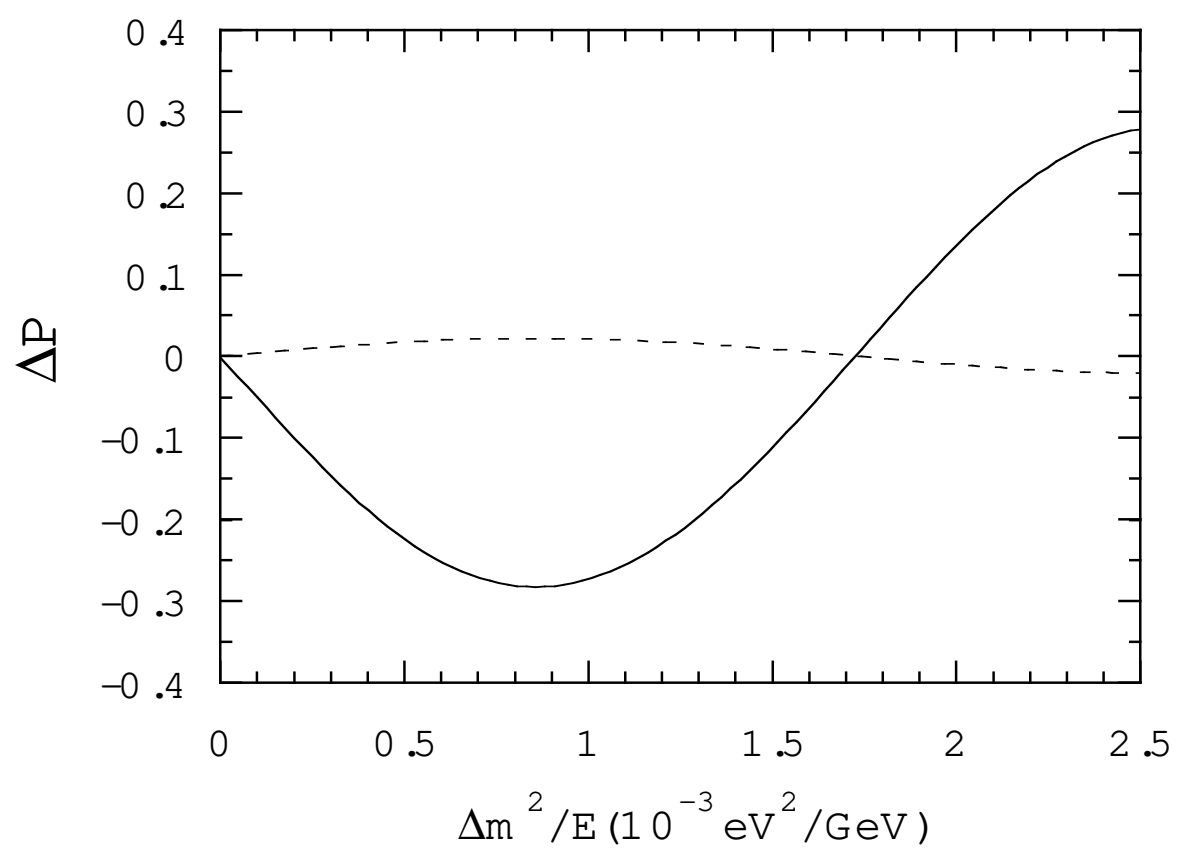

Fig. 8 


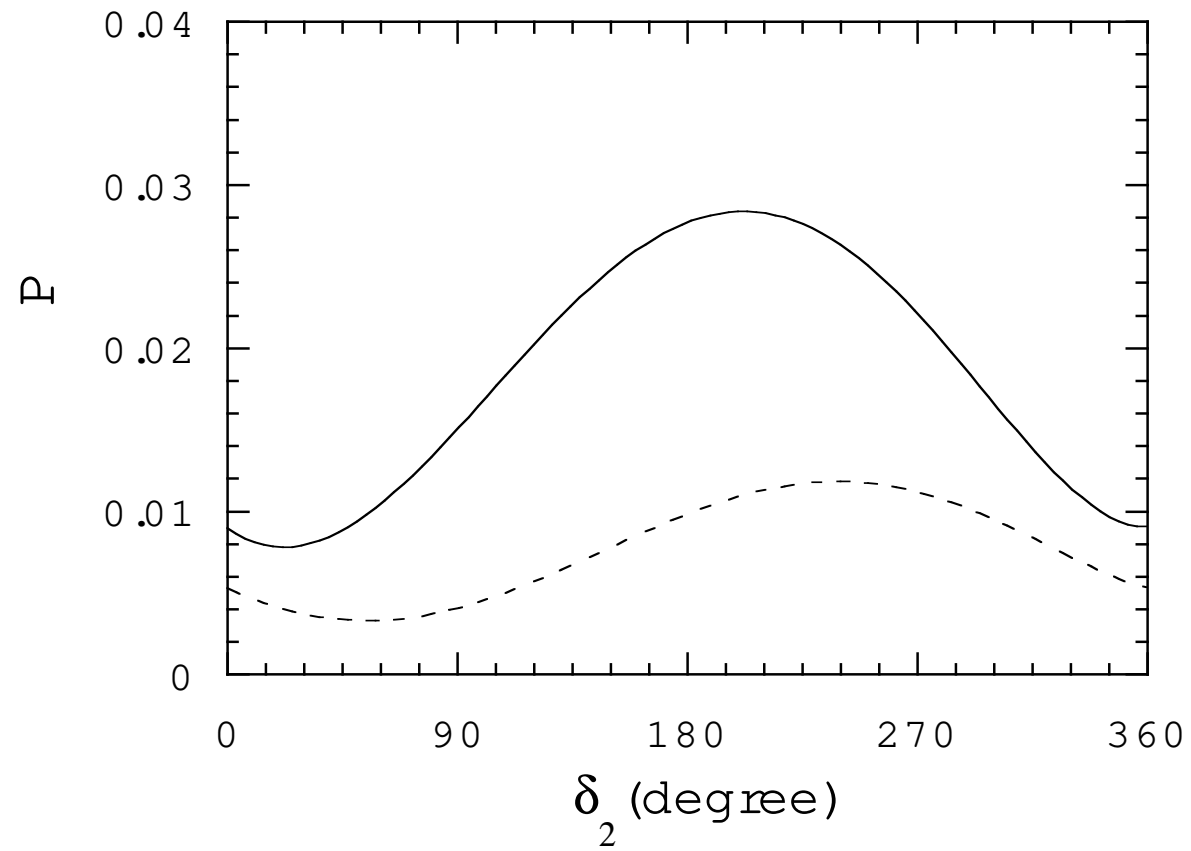

Fig. 2 


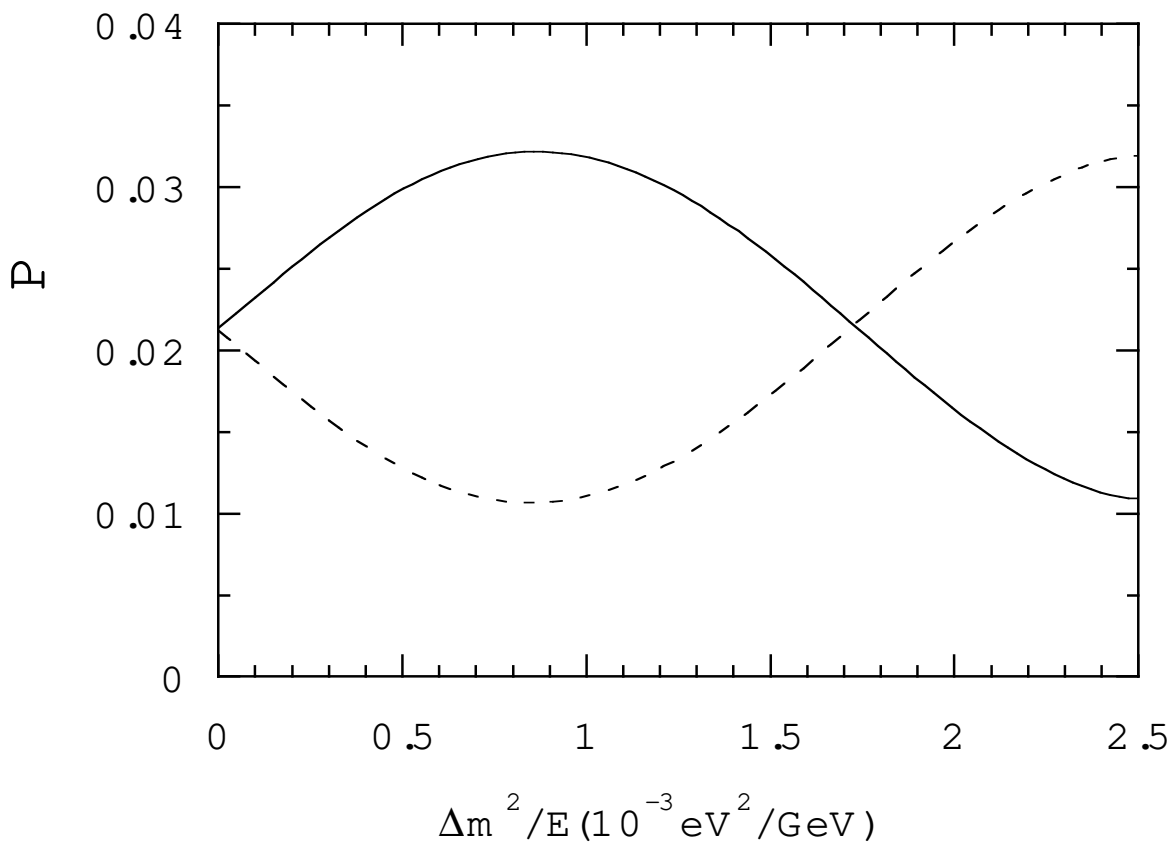

Fig. 9 


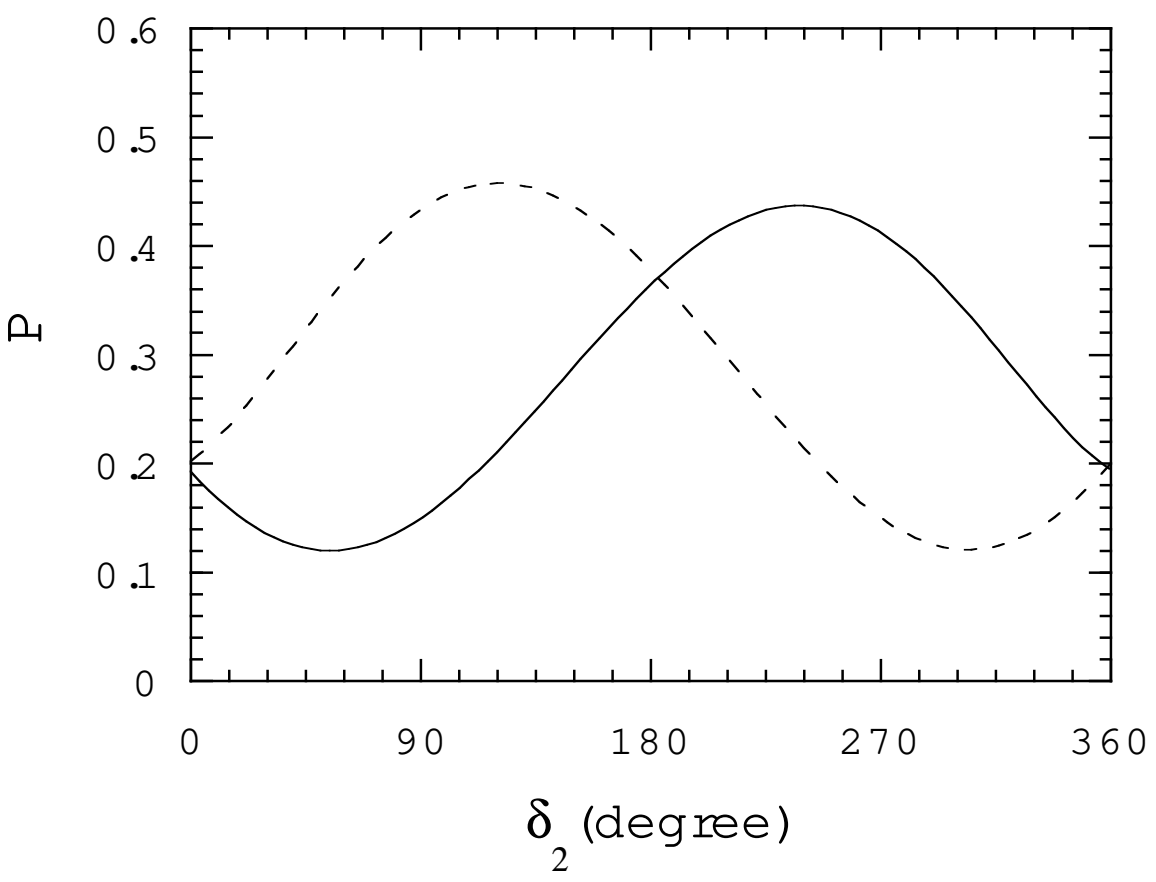

Fig . 4 


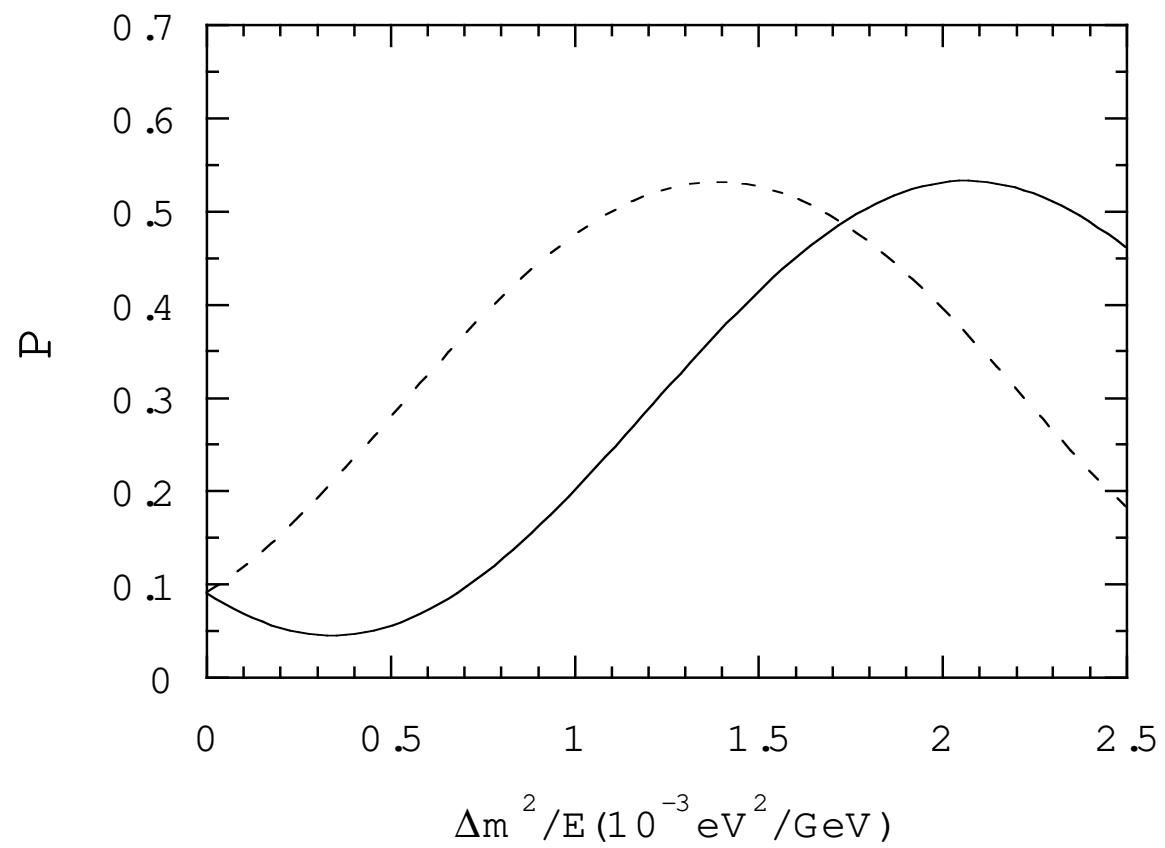

Fig. 7 


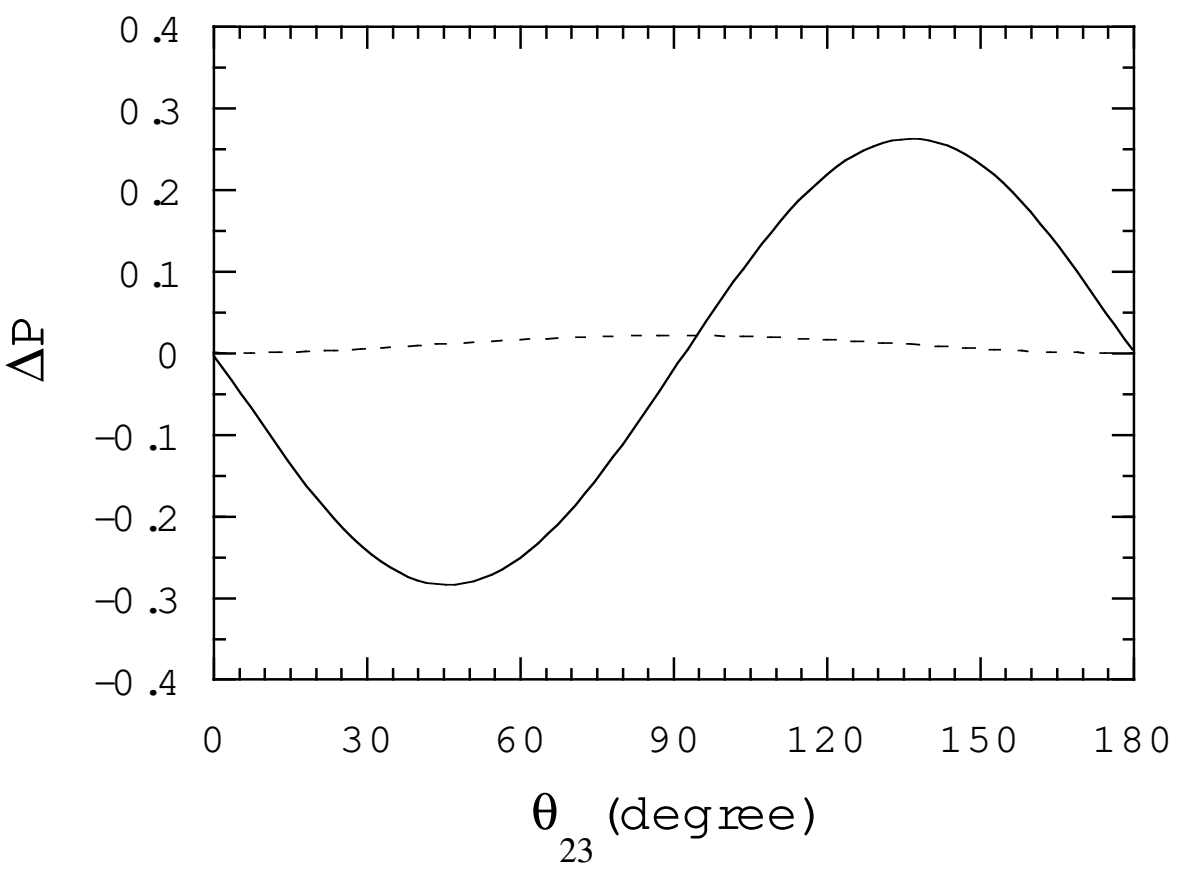

Fig. 6 